\title{
Review of Gait Analysis and Planning of Legged Robot
}

\author{
Jiahan $\mathrm{Li}^{1, \mathrm{a}^{*}}$, Ying Sun ${ }^{1,2, \mathrm{~b}}$ and $\mathrm{Hao} \mathrm{Wu}^{1, \mathrm{c}}$ \\ ${ }^{1}$ Key Laboratory of Metallurgical Equipment and Control Technology, Ministry of Education, Wuhan \\ University of Science and Technology, Wuhan 430081, China \\ ${ }^{2}$ Hubei Key Laboratory of Mechanical Transmission and Manufacturing Engineering, Wuhan \\ University of Science and Technology, Wuhan 430081, China \\ ajiahan.li@foxmail.com, bsunying65@wust.edu.cn, c1252334436@qq.com \\ * please mark the corresponding author with an asterisk
}

Keywords: Legged Robot; Adaptation; Gait analysis; Gait planning; Kinematics

\begin{abstract}
Legged robot is more adaptable to complex environment than wheeled or tracked robot. Therefore, legged robot has attracted much attention. Being inspired by the movements of man and animals, the main concern is biped robots, quadruped robot and hexapod robot. Gait analysis and planning are the keys to legged robot walking. At present, there are many researches on gait of legged robot and a lot of achievements have been obtained. These researches have effectively promoted the development of gait analysis and planning.
\end{abstract}

\section{Introduction}

The concept of legged robot is mainly based on the uneven terrain changes in nature. In the irregular and uneven terrain environment, multiped showed good ability to adapt to harsh terrain environment. Wheeled or tracked vehicle can't reach nearly half of the earth surface. But the multiped can freely walk in these places [1]. Comparison of legged robot and wheeled or tracked robot. It can pass irregular and loose terrain while the body remains stable. It can cross obstacles, trenches, and stairways. It also can move in all directions finally [2].

Because of the advantages of legged robot, legged robot has become a hot spot in the field of bionic robot in recent years. The research of biped robot is mainly focused on biped robot, quadruped robot and hexapod robot. One of the most important fields of robot research is motion planning and analysis of robots. For the biped robot, it is robot gait planning and control strategies.

The motion of a legged robot is accomplished mainly by mechanical leg. To judge the performance of a robot, its motion performance is an important criterion. The motion of a mechanical leg is described by gait. In this paper, I will introduce the research situation of gait analysis and planning of several hot legged robots.

\section{Important Analysis Parameters of Gait Analysis}

Legged robot gait refers to the movement of each leg in a certain order and trajectory. This movement realizes the robot's walking movement. The time required for a robot to complete a gait is called a gait cycle. A complete gait process is the movement of all the legs to complete a "lift, swing, release" movement. During this process, the robot body also completes the transition process. The gait cycle parameters of the legged robot are adjustable, and the parameters of the gait cycle are limited by the performance of the actuator.

The degree of freedom of motion of the robot body is calculated as Eq. 1 In the robot gait analysis, there is an important technical parameter is the area coefficient. The area coefficient is the ratio of the time each foot touches the ground to the entire gait cycle. When the area coefficient is equal to 0.5 , The robot alternately swings two sets of legs. This gait known as trot. When the area coefficient is less than 0.5 , the robot has only three legs to support the ground at every moment, which is called jumping gait. When the area coefficient is greater than 0.5 , the robot turns over three feet to support the ground. This gait is known as slow crawl gait. The area coefficient can be used to 
describe the motion activity of a legged robot in a mathematical way [3].

$$
f_{0}=\lambda(n-j-1)+\sum f_{i} .
$$

Stride length is also an important analysis parameter for gait analysis of legged Robot. The definition of robot stride length is the translation of the center of gravity of a robot in a gait cycle. A perfect analysis of a legged robot requires an analysis of the static performance of the robot. The static performance of legged robot is closely related to gait generation strategy. The gait generation strategy determines the walking stability of the robot. Stability analysis is necessary for gait generation. For multi legged robot, there must be enough legs based on ground support robot at any time, it can ensure the static stability of walking. Usually, at least three legs are required, and the triangle made up of the three legs must be surrounded by a vertical projection of the center of gravity of the robot.

The above parameters are the most important parameters that determine the gait of a robot. These parameters determine the gait generation strategy of a legged robot.

\section{Gait Analysis and Planning of Biped Robot}

There are two main kinds of gait planning methods for biped robot: bionic method based on human walking data (HMCD) and the planning method which based on stability [4]. The latter one is typically a gait planning method based on the stability criterion of zero-moment point (ZMP) [5]. Some researchers have researched gait planning using ZMP method [6]. ZMP is an important basic research of biped robot gait planning.

At present, most of the motion planning of biped robot is assumed that the ground is ideal rigid. However, the gait planning research in the ideal laboratory environment has a great negative impact on the environmental adaptability of biped robot. Therefore, many scholars begin to consider the influence of walk path viscoelasticity on robot gait parameters for the walk problem in natural ground environment [7]. Some researchers begin to study the dynamic walk control of biped robot with uneven ground, and adopt the linear inverted pendulum model and the concept of energy conservation to generate global variables [8]. Through the design of swing foot and centroid trajectory of biped robot to solve gait. The centroid of biped robot is considered as a linear inverted pendulum. The trajectory of the end of the swinging leg is represented by a smoothing function that connects the landing points of the two sampling times [9].

But the above research ignores the flexibility of walking path. According to the coupling dynamics analysis between robot and operating path, the most typical flexible path environment is sand, and in the complex environment, such as sand, the normal operation is also an important development trend of biped robot. Based on this condition, some researchers begin to research the driving wheels and attachments of the sand robot, and put forward a combination of rigidity and flexibility, which is a reasonable method of gait analysis [10]. Some researchers have studied the multi body dynamics simulation of the volcano detection robot for the special environment of the volcano detection. In this research, the contact coupling characteristics between 6 walking wheels and the ground have been considered [11]. At the same time, the dynamic characteristics of the wheeled robot during the operation of the flexible cable in the process of rolling, obstacle avoidance and attitude adjustment are analyzed [12]. Although this research is aimed at the research of wheeled robot's working process, it is also a good guide for kinematics research of legged robot in flexible trajectory. From the above, when the biped robot's gait planning and dynamics are studied it is found that the influence of surface viscoelasticity on the dynamic response and walking stability of biped robot has not been systematically analyzed.

\section{Gait Analysis and Planning of Quadruped Robot}

In order to realize the stable walking of quadruped robot, it is necessary to analyze the kinematics and plan the gait of the quadruped robot firstly. Quadruped robot gait planning refers to motion 
trajectory planning of robot walking process [13-14]. The joint space trajectory planning, planning function generated value is the amount of joint and Cartesian space trajectory planning, function generation is the value of the robot end effector pose, they need by solving the inverse kinematics equation can be transformed into joint volume. In the joint space trajectory planning, the planning function calculation is joint, and the cartesian space trajectory planning function calculation is the robot end-effector pose, the calculation need to be solved by the inverse kinematic equations can be transformed into joint volume. According to the process of walking each leg movement order and the different way of quadruped robot gait can be divided into walking gait, amble gait, diagonal gait, jogging gait, running jogging, jumping gait [15].

In the research process of quadruped robot, the method of static walking is realized firstly. Every time when running only one leg in the swing phase, in support of the other three legs. The robot can remain stable as long as the projection of the center of gravity of the robot in the support plane at any given moment falls in the triangular region of the three support legs. But the coefficient of the duty ratio of the static walk is large, and it requires four legs to swing in a certain order to complete a walking cycle movement. Compared with the static walking mode, the gait with diagonal gait is more efficient and energy-saving. Therefore, this gait is widely used to realize the high-speed motion of quadruped robot, and it is feasible and practical. The technique of dynamic walking is much more difficult than that of static walking. Therefore, in the process of walking with diagonal gait, how to smoothly enter the stable running state from the starting state and prevent the robot from tipping over and ensuring its stability when walking is the key problem that needs to be solved urgently.

\section{Gait Analysis and Planning of Hexapod Robot}

As the core of the basic theory research of hexapod robot, gait planning provides theoretical basis for trajectory planning and motion planning. It determines the order of each foot movement, and directly affects the overall performance of the robot. It is the key for the hexapod robot to efficiently pass through the complex unstructured environment and give full play to its potential. The experimental observations show that the creatures use periodic rhythmic gaits in flat terrain, whereas in rugged terrain they use free gaits [16]. Therefore, the gait planning of hexapod robot is mainly aimed at these two types of gait.

When hexapod creatures walk on a flat terrain, each foot rises and falls approximately periodically in a particular order. Its gait can be described by $\beta_{i}$ and $\phi_{i}$. As shown in Figure $1, \beta_{i}$ is the duty cycle of $\mathrm{i}$ feet, and $\phi_{i}$ is the relative phase of $\mathrm{i}$ feet. That is the ratio of the touchdown time of a foot $i$ to the touchdown time of reference foot in a gait cycle. If the two gait parameters of hexapod robot are a fixed value, it will form a regular periodic gait, if these two parameters take different values, will form a regular periodic gait rich, such as triangle gait, quadrupedal gait and waveform gait [17].

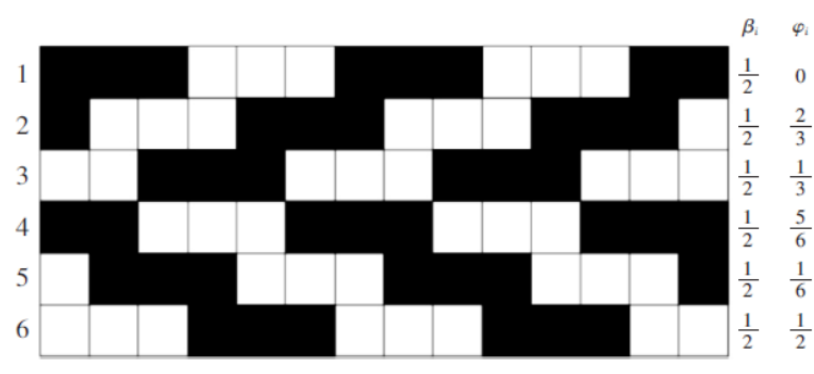

Figure 1. Finite Gait map of hexapod robot

Triangle gait is one of the most widely used gaits in hexapod robots [18]. During the movement, moving from side of the front foot, post foot structure and the other side of the midfoot support alternate robot swing. The triangle gait is not only simple to control, but it is also a very efficient 
gait [19]. Quadruped gait is a relatively slow gait. At any moment, the robot is supported by four limbs, and both sides of the robot have one foot to swing forward, so the quadruped gait can bear greater load [20-21]. The waveform gait has five feet at each moment in the supporting phase, and the six feet move in a certain order, so the speed of the waveform gait are slow.

Free gait is a better gait, corresponding to the above gait. The free gait can adjust the gait parameters, such as area factor and relative phase, in each gait cycle. It makes the robot can speed, turn, obstacle avoidance, obstacle crossing and other purposes, so that the hexapod robots have more outstanding sports potential [22]. Therefore, based on local rules and CPG principle, a free gait planning method is proposed based on different angles to simulate biological gait. These gait planning methods basically includes the most common and widely used hexapod walking machine planning method at present.

\section{Conclusions}

In this paper, the gait planning and analysis methods of some main legged robots are discussed. Some suggestions are given to improve motion performance and gait planning and analysis methods of legged robot. Generally speaking, the existing research has made a breakthrough in the design of legged robot, bionic structure and coordinated control of multi legged gait planning. A lot of robot prototypes are developed, and the bionic movement of the robot is realized. But at present, there are still many problems in legged robot, such as walking too mechanically, unable to finish complex bionic movement, and poor terrain adaptability. Its bionic degree is relatively primary. There is still a lot of space for growth in this field.

\section{References}

[1] B.Lofterod, T.Terjesen and I.Skaaret:Acta Orthopaedica. Vol. 78 (2007) No.1, p.74.

[2] E.Garcia, M.A.Jimenez and P.G.Santos:IEEE Robotics and Automation Society. Vol. 4 (2007) No.1, p.90.

[3] T.Wren, G.Gorton and C.Tuker: Gait and Posture. Vol. 34 (2011) No.2, p.149.

[4] X.Chen, L.Wang and X.Ye: Mechatronics. Vol. 23 (2013) No.4, p.429.

[5] M.Vukobratovic, B.Brovac : Journal of Humanoid Robotics. Vol. 1 (2004) No.1, p.157.

[6] V.Cimolin, M.Galli: Gait and Posture. Vol. 39 (2014) No.4, p.1005.

[7] Z.L.Wang, G.C.Yu and L.Jie: Computer Science. Vol. 32 (2010) No.12, p.215.

[8] S.Kajita, T.Nagasaki and K.Kaneko: Proceedings of the 2005 IEEE International Conference on Advanced Robotics. (Barcelona, Spain, 2005). p.616.

[9] Y.Fujimoto, I.A.Smadi and Y Wakayama: Mechatronics(ICM), IEEE International Conference. (2011). p.821.

[10] Y.L.Fu, H.Xu and S.G.Wang: Robot technology and Application. Vol. 4 (2004), p.22.

[11] L.Michele, M.Giovanni and S.Rosario: IEEE International Conference on Intelligent Robots and Systems. (Epfl, Switzerland, 2002). p.714.

[12]X.H.Xiao, G.P.Wu and E.Du: Journal of Center South University of Technology. Vol. 15 (2008) No.6, p.869.

[13] O.Tirosh, R.Baker and J.McGinley : Computers in Biology and Medicine. Vol. 40 (2010) No.2, p.201.

[14] D.Q.Hu, P.S.Ma : Computer Simulation. Vol. 22 (2005) No.2, p.147.

[15] W.Tao, R.C.Zhang and H.T.Feng :Sensors. Vol. 12 (2012) No.2, p.2255.

[16]L.Huang, B.L.Han and Q.S.Luo : Journal of Huazhong University of Science and Technology. Vol. 35 (2007) No.12, p.72.

[17] G.Wang, L.X.Zhang and L.Q.Wang : Journal of Harbin Engineering University. Vol. 32 (2011) No.4, p.486.

[18] J.M.Porta, E.Celaya : Robotics and Autonomous Systems. Vol. 47 (2004), p.187.

[19]P.Chen, Z.X.Zhang and J.Zhao : Machinery and Electronics. Vol. 32 (2009) No.9, p.53.

[20] P.S.Cristina, M.Vitor : Robotics and Autonomous Systems. Vol. 59 (2011) No.9, p.620. 
[21]P.S.Cristina, M.Vitor : Robotics and Autonomous Systems. Vol. 60 (2012) No.6, p.912.

[22] M.S.Erden, L.Kemal : Robotics and Autonomous Systems. Vol. 56 (2008) No.3, p.199. 